\title{
Shadow Bounds for Self-Dual Codes
}

\author{
Eric M. Rains
}

\begin{abstract}
Conway and Sloane have previously given an upper bound on the minimum distance of a singly-even self-dual binary code, using the concept of the shadow of a self-dual code. We improve their bound, finding that the minimum distance of a selfdual binary code of length $n$ is at most $4\lfloor n / 24\rfloor+4$, except when $n \bmod 24=22$, when the bound is $4\lfloor n / 24\rfloor+6$. We also show that a code of length a multiple of 24 meeting the bound cannot be singly-even. The same technique gives similar results for additive codes over GF (4) (relevant to quantum coding theory).
\end{abstract}

Index Terms-Bound, self-dual code, shadow, singly-even.

\section{INTRODUCTION}

$\mathbf{I}$ $\mathrm{N}$ [5], the following was shown:

Theorem: If a doubly-even self-dual $[n, n / 2, d]$ exists, then $d \leq 4\left\lfloor\frac{n}{24}\right\rfloor+4$.

The objective of the present work is to remove the restriction that the code be doubly-even. For singly-even codes, much less has hitherto been known; a direct extension of the proof in [5] gives a bound $d \leq 2\left\lfloor\frac{n}{8}\right\rfloor+2$, but this bound is almost never met. The situation was improved greatly by [2], which gives a bound $d \leq 2\left\lfloor\frac{n+6}{10}\right\rfloor$, except when $n$ is $2,12,22$, or 32 ; a further improvement appears in [7], which gives the bound $d \leq(n / 6)+2+(2 / 3) \log _{2}(n)$. This is still higher than the bound for doubly-even codes, however. In the sequel, a new bound is proved, of the form

$$
d \leq 4\left\lfloor\frac{n}{24}\right\rfloor+4
$$

except when $n \bmod 24=22$, when

$$
d \leq 4\left\lfloor\frac{n}{24}\right\rfloor+6 \text {. }
$$

In particular, whenever $n$ is a multiple of 8 , so both singlyeven and doubly-even codes exist, we now have the same bound for singly-even and doubly-even codes. In fact, when $n$ is a multiple of 24 , it can be shown that any code meeting the bound must be doubly-even.

As the present bound is shown using linear programming, it is natural to inquire how much weaker it is than the full LP bound. Using a high-precision LP package (the author used maple), one can readily verify that for all $n$ in the range $8 \leq n \leq 200$, there exists a feasible weight enumerator (including the constraints from the shadow enumerator (see below)) meeting the bound. In some cases, the present bound can be improved upon using integer programming, however.

The key idea in the proof is the use of additional constraints coming from the "shadow" of the code [2]. It turns out that

Manuscript received January 21, 1997; revised June 20, 1997.

The author is with AT\&T Labs-Research, Florham Park, NJ 07932-0971 USA.

Publisher Item Identifier S 0018-9448(98)00087-X. this concept has a natural analog in the case of additive codes over GF (4); that is, GF (2)-linear subsets of GF (4) ${ }^{n}$, selforthogonal (i.e., contained in its dual) under the inner product

$$
\langle v, w\rangle=\sum_{i} \operatorname{Tr}\left(v_{i}^{2} w_{i}\right) .
$$

These codes appear, for instance, in the theory of quantum error-correcting codes [1]. For these codes, we give a bound $2\left\lfloor\frac{n}{6}\right\rfloor+2$, or $2\left\lfloor\frac{n}{6}\right\rfloor+3$ when $n \bmod 6=5$. We also give a result bounding the minimum weight of $C^{\perp}-C$ when $C$ is a self-orthogonal additive code.

A quick note on notation: We will use the notation $[n, k, d]_{4}$ to refer to an additive code over GF $(4) ; k$ will be its dimension as a vector space over GF (2). In particular, a self-dual code will have $k=n$.

\section{SHADOWS}

Let $C$ be a self-orthogonal binary code. From the congruence

$$
\mathrm{wt}(v+w)-\mathrm{wt}(v)-\mathrm{wt}(w) \equiv 2\langle v, w\rangle \equiv 0(\bmod 4)
$$

it follows that the subset of $C$ consisting of elements of weight a multiple of 4 forms a subspace $C_{0}$ of $C$. If $C$ is doublyeven, then $C_{0}=C$, and we define the shadow $S(C)=C^{\perp}$. Otherwise, we define $S(C)=C_{0}^{\perp}-C^{\perp}$. Equivalently, $S(C)$ is the set of vectors $w$ such that

$$
2\langle w, v\rangle \equiv \operatorname{wt}(v)(\bmod 4)
$$

for all $v \in C$.

Theorem 1: Let $A(x, y)$ be the weight enumerator of $C$, and let $S(x, y)$ be the weight enumerator of $S(C)$. Then

$$
S(x, y)=\frac{1}{|C|} A(x+y, i(x-y)) .
$$

Proof: See [2, Theorem 6, in particular, eq. (23)]. Note that [2] considers codes containing their duals, rather than codes contained in their duals; thus one should exchange $C$ and $C^{\perp}$ throughout.

Similarly, let $C$ be an additive code over GF (4), selforthogonal under the above inner product. One can readily verify that

$$
\mathrm{wt}(v+w)-\mathrm{wt}(v)-\mathrm{wt}(w) \equiv\langle v, w\rangle \equiv 0 \bmod 2
$$

so as above, the subset $C_{0}$ of even codewords in $C$ is a subgroup; defining $S(C)$ as above, or equivalently, as the set of vectors $w$ such that

$$
\langle v, w\rangle \equiv \mathrm{wt}(v) \bmod 2
$$

for all $v \in C$, we have 
Theorem 2: Let $A(x, y)$ be the weight enumerator of $C$, and let $S(x, y)$ be the weight enumerator of $S(C)$. Then

$$
S(x, y)=\frac{1}{|C|} A(x+3 y, y-x) \text {. }
$$

Proof: Completely analogous.

For self-dual codes, the weight enumerators have a special form, which carries over to the shadow enumerator

Theorem 3: Let $A(x, y)$ and $S(x, y)$ be, respectively, the enumerator of a self-dual binary code of length $n$ and that of its shadow. Then there exist coefficients $c_{i}, 0 \leq i \leq\left\lfloor\frac{n}{8}\right\rfloor$, such that

$$
\begin{aligned}
& A(x, y)=\sum_{0 \leq i \leq\lfloor n / 8\rfloor} c_{i}\left(x^{2}+y^{2}\right)^{n / 2-4 i}\left\{x^{2} y^{2}\left(x^{2}-y^{2}\right)^{2}\right\}^{i} \\
& S(x, y)=\sum_{0 \leq i \leq\lfloor n / 8\rfloor}(-1)^{i} c_{i} 2^{n / 2-6 i}(x y)^{n / 2-4 i}\left(x^{4}-y^{4}\right)^{2 i} .
\end{aligned}
$$

Proof: This is part 4 of [2, Theorem 5].

Analogously, we have

Theorem 4: Let $A(x, y)$ and $S(x, y)$ be, respectively, the enumerator of a self-dual additive code over GF (4) of length $n$ and that of its shadow. Then there exist coefficients $c_{i}, 0 \leq$ $i \leq\left\lfloor\frac{n}{2}\right\rfloor$, such that

$$
\begin{aligned}
& A(x, y)=\sum_{0 \leq i \leq\lfloor n / 2\rfloor} c_{i}(x+y)^{n-2 i}(y(x-y))^{i} \\
& S(x, y)=\sum_{0 \leq i \leq\lfloor n / 2\rfloor}(-1)^{i} 2^{n-3 i} c_{i} y^{n-2 i}\left(x^{2}-y^{2}\right)^{i} .
\end{aligned}
$$

Proof: Analogous.

In each case, we prove our bound by expressing an appropriately chosen $c_{i}$ both as a linear combination of the initial coefficients of the weight enumerator and as a linear combination of the initial coefficients of the shadow enumerator. All but one of the terms in the first linear combination will be 0 , based on the putative minimum distance; consequently, the first linear combination reduces to an explicit constant. All coefficients in the second linear combination will turn out to have the same sign, a sign inconsistent with the sign of $c_{i}$.

\section{BINARY CODES}

Let $C$ be a self-dual binary code, with shadow $S$; let $A(x, y)$ and $S(x, y)$ be the respective weight enumerators. Write, as in Theorem 3,

$$
\begin{aligned}
A(1, y) & =\sum_{0 \leq j \leq\lfloor n / 2\rfloor} a_{j} y^{2 j} \\
& =\sum_{0 \leq i \leq\lfloor n / 8\rfloor} c_{i}\left(1+y^{2}\right)^{n / 2-4 i}\left(y^{2}\left(1-y^{2}\right)^{2}\right)^{i} \\
S(1, y) & =\sum_{0 \leq j \leq 2\lfloor n / 8\rfloor} b_{j} y^{4 j+t} \\
& =\sum_{0 \leq i \leq\lfloor n / 8\rfloor}(-1)^{i} c_{i} 2^{n / 2-6 i} y^{n / 2-4 i}\left(1-y^{4}\right)^{2 i}
\end{aligned}
$$

where $t=(n / 2 \bmod 4)$. Note that $a_{0}=1$, and all $a_{j}$ and $b_{j}$ must be nonnegative integers. Also, one can write $c_{i}$ as a linear combination of the $a_{j}$ for $0 \leq j \leq i$, and as a linear combination of the $b_{j}$ for $0 \leq j \leq\lfloor n / 8\rfloor-i$.

Define $\alpha_{i}(n)$ to be the coefficient of $a_{0}$ in the expansion of $c_{i}$ in terms of $a_{j}$ for $0 \leq j \leq i$, and define $\beta_{i j}(n)$ to be the coefficient of $b_{j}$ in the expansion of $c_{i}$ in terms of $b_{j}$ for $0 \leq j \leq\lfloor n / 8\rfloor-i$. Then, except in extreme cases, we will see that $\alpha_{i}(n)<0$ for suitably chosen $i$, while $\beta_{i j}(n)>0$ for the same $i$ and $0 \leq j \leq\lfloor n / 8\rfloor-i$. Thus we need to compute $\alpha_{i}(n)$ and $\beta_{i j}(n)$ at strategically chosen points.

First, $\alpha_{i}(n)$. For $i>0$

$\alpha_{i}(n)=-\frac{n}{2 i}\left[\right.$ coeff. of $y^{i-1}$ in $\left.(1+y)^{-(n / 2)-1+4 i}(1-y)^{-2 i}\right]$.

This is [2, eq. (48)], and follows from the Bürmann-Lagrange theorem:

Theorem (Bürmann-Lagrange): Let $f(x)$ and $g(x)$ be formal power series, with $g(0)=0$, and $g^{\prime}(0) \neq 0$. If coefficients $\kappa_{i j}$ are defined by

$$
x^{j} f(x)=\sum_{0 \leq i} \kappa_{i j} g(x)^{i}
$$

then

$\kappa_{i j}=\frac{1}{i}\left[\right.$ coeff. of $x^{i-1}$ in $\left.\left[j x^{j-1} f(x)+x^{j} f^{\prime}(x)\right]\left(\frac{x}{g(x)}\right)^{i}\right]$.

Proof: See [8, ch. 7].

In particular, for $l \geq 1$, we have

$$
\begin{aligned}
& \alpha_{2 m}(24 m-2 l)=-\frac{12 m-l}{2 m}\left[\operatorname{coeff} . \text { of } y^{2 m-1}\right. \text { in } \\
&\left.(1+y)^{-4 m+l-1}(1-y)^{-4 m}\right] \\
&=-\frac{12 m-l}{2 m}\left[\text { coeff. of } y^{2 m-1}\right. \text { in } \\
&\left.(1+y)^{l-1}\left(1-y^{2}\right)^{-4 m}\right] \\
&= \\
& \\
&\left(\begin{array}{c}
l-1 \\
k
\end{array}\right)\left(\begin{array}{c}
(10 m-k-3) / 2 \\
4 m-1
\end{array}\right) .
\end{aligned}
$$

For $1 \leq l \leq 13$ and $m \geq 2$, each term in the sum is nonnegative, so we can conclude that $\alpha_{2 m}(24 m-2 l) \leq 0$, with equality only when $l=1$. Similarly, $\alpha_{2 m+1}(24 m-2)<0$ (we need this to handle $n \bmod 24 \equiv 22$ ).

We will need two more values of $\alpha$ to handle the case $n \bmod 24=0$ (i.e., to show that a self-dual $[24 m, 12 m$, $4 m+4]$ must be doubly-even):

$$
\begin{aligned}
\alpha_{2 m}(24 m)= & -6\left[\text { coeff. of } y^{2 m-1}\right. \text { in } \\
& \left.(1+y)^{-4 m-1}(1-y)^{-4 m}\right] \\
= & -6\left[\text { coeff. of } y^{2 m-1} \text { in }(1-y)\left(1-y^{2}\right)^{-4 m-1}\right] \\
= & 6\left[\text { coeff. of } z^{m-1} \text { in }(1-z)^{-4 m-1}\right] \\
= & 6(-1)^{m-1}\left(\begin{array}{c}
-4 m-1 \\
m-1
\end{array}\right) \\
= & \frac{6}{5}\left(\begin{array}{c}
5 m \\
m
\end{array}\right)
\end{aligned}
$$


and

$$
\begin{aligned}
& \alpha_{2 m+1}(24 m)=-\frac{12 m}{2 m+1}\left[\text { coeff. of } y^{2 m}\right. \text { in } \\
&\left.(1+y)^{-4 m+3}(1-y)^{-4 m-2}\right] \\
&=-\frac{12 m}{2 m+1}\left[\operatorname{coeff} \text { of } y^{2 m}\right. \text { in } \\
&\left.(1+y)^{5}\left(1-y^{2}\right)^{-4 m-2}\right] \\
&=-\frac{12 m}{2 m+1} \sum_{0 \leq k \leq 2}\left(\begin{array}{c}
5 \\
2 k
\end{array}\right)(-1)^{m-k} \\
& \cdot\left(\begin{array}{c}
-4 m-2 \\
m-k
\end{array}\right) \\
&=-192 \frac{m^{2}}{(2 m+1)(4 m+1)}\left(\begin{array}{c}
5 m \\
m
\end{array}\right) .
\end{aligned}
$$

It will turn out that

$$
\alpha_{2 m}(24 m)=\beta_{(2 m) 0}(24 m)
$$

and

$$
\alpha_{2 m+1}(24 m)=\beta_{(2 m+1) 0}(24 m)
$$

A similar Bürmann-Lagrange calculation gives a formula for $\beta_{i j}(n)$

$$
\beta_{i j}=(-1)^{i} 2^{-n / 2+6 i} \frac{k-j}{i}\left(\begin{array}{c}
k+i-j-1 \\
k-i-j
\end{array}\right)
$$

valid for $i>0$, where $k=\lfloor n / 8 \mid$. Note, in particular, that $(-1)^{i} \beta_{i j}>0$ for $0 \leq j \leq k-i$. The details are omitted for conciseness; the calculation is essentially that in [2], except for an error in [2, eq. (55)] (the second term should be added, not subtracted).

We can now prove

Theorem 5: If a self-dual $[24 m+2 l, 12 m+l, d]$ exists, with $0 \leq l \leq 11$, then

$$
d \leq \begin{cases}4 m+4, & l<11 \\ 4 m+6, & l=11 .\end{cases}
$$

If a self-dual $[24 m+22,12 m+11,4 m+6]$ exists, then so does a doubly-even self-dual $[24 m+24,12 m+12,4 m+8]$. Finally, any self-dual $[24 m, 12 m, 4 m+4]$ must be doubly-even.

Proof: We first show that $d \leq 4 m+4$ for $0 \leq l<11$. Suppose, on the contrary, that $d>4 m+4$. Consider $c_{2 m+2}$. On the one hand, $c_{2 m+2}$ is $\alpha_{2 m+2}(n)$ plus a linear combination of the $a_{i}$ for $1 \leq i \leq 2 m+2$; since these are all 0 , we have

$$
c_{2 m+2}=\alpha_{2(m+1)}(24(m+1)-2(12-l))<0 .
$$

On the other hand,

$$
c_{2 m+2}=\sum_{j} \beta_{(2 m+2) j} b_{j} .
$$

But $\beta_{(2 m+2) j} b_{j}$ is nonnegative for all $j$. So $c_{2 m+2} \geq 0$, a contradiction.

Now, consider a self-dual $[24 m+22,12 m+11,4 m+6]$. In this case, we have

$\sum_{0 \leq j \leq m} \beta_{(2 m+2) j} b_{j}=c_{2 m+2}=\alpha_{2(m+1)}(24(m+1)-2)=0$.
But then $b_{j}=0$ for $0 \leq j \leq m$. In other words, the shadow code must have minimum weight $4 m+6$ as well. Letting $C^{(i)}$ for $0 \leq i \leq 3$ be the four cosets of the even subcode $C^{(0)}$ in its dual, we can construct an even self-dual $[24 m+24,12 m+12,4 m+8]$ as the set of all vectors of one of the following four forms: $(0,0) \mid v$, for $v \in C^{(0)},(0,1) \mid v$, for $v \in C^{(1)},(1,0) \mid v$, for $v \in C^{(3)}$, or $(1,1) \mid v$, for $v \in C^{(2)}$. (This construction is given in [4].)

The possibility of a self-dual $[24 m+22,12 m+11,4 m+$ 8] can be eliminated by remarking that $c_{2 m+3}$ is a linear combination of $b_{j}$ for $0 \leq j<m$, so must be 0 , but

$$
c_{2 m+3}=\alpha_{2(m+1)+1}(24(m+1)-2)<0 .
$$

Finally, consider a putative $[24 m, 12 m, 4 m+4]$. Consider

$$
F=\alpha_{2 m+1}(24 m) c_{2 m}-\alpha_{2 m}(24 m) c_{2 m+1} \text {. }
$$

Since

$$
\alpha_{2 m+1}(24 m)=\beta_{(2 m+1) 0}(24 m)
$$

and

$$
\alpha_{2 m}(24 m)=\beta_{(2 m) 0}(24 m)
$$

$F$ is a linear combination of $a_{1}$ through $a_{2 m+1}$, so must be 0 . On the other hand, we have

$$
\begin{aligned}
F & =\alpha_{2 m+1}(24 m) c_{2 m}-\alpha_{2 m}(24 m) c_{2 m+1} \\
& =\sum_{0 \leq j \leq m} \beta_{(2 m+1) 0} \beta_{(2 m) j} b_{j}-\beta_{(2 m) 0} \beta_{(2 m+1) j} b_{j} \\
& =-\sum_{0 \leq j \leq m} b_{j}\left(\frac{384}{5} \frac{j(3 m-j)(6 m-j)}{(2 m+1)(4 m+1)(5 m-j)}\right. \\
& \left.\cdot\left(\begin{array}{c}
5 m \\
m
\end{array}\right)\left(\begin{array}{c}
5 m-j \\
m-j
\end{array}\right)\right) .
\end{aligned}
$$

This is a negative linear combination of $b_{1}$ through $b_{m}$. In other words, $b_{1}$ through $b_{m}$ must all be 0 . But then

$$
\beta_{(2 m+1) 0}=\alpha_{2 m+1}(24 m)=c_{2 m+1}=\beta_{(2 m+1) 0} b_{0}
$$

so $b_{0}=1$. But this can only happen if the code is doubly even.

\section{Additive Codes Over GF (4)}

Let $C$ be a self-dual additive code over GF(4), with shadow $S$; let $A(x, y)$ and $S(x, y)$ be the respective weight enumerators. Write, as in Theorem 4 ,

$$
\begin{aligned}
A(1, y) & =\sum_{0 \leq j \leq n} a_{j} y^{j} \\
& =\sum_{0 \leq i \leq\lfloor n / 2\rfloor} c_{i}(1+y)^{n-2 i}(y(1-y))^{i} \\
S(1, y) & =\sum_{0 \leq j \leq\lfloor n / 2\rfloor} b_{j} y^{2 j+t} \\
& =\sum_{0 \leq i \leq\lfloor n / 2\rfloor}(-1)^{i} 2^{n-3 i} c_{i} y^{n-2 i}\left(1-y^{2}\right)^{i}
\end{aligned}
$$

where $t=(n \bmod 2)$. As before, $a_{0}=1,0 \leq a_{j}, b_{j}$, and $c_{i}$ can be written either as a linear combination of the $a_{j}$ 
for $0 \leq j \leq i$, or as a linear combination of the $b_{j}$ for $0 \leq j \leq\lfloor n / 2\rfloor-i$.

Define $\alpha_{i}(n)$ to be the coefficient of $a_{0}$ in $c_{i}$; define $\beta_{i j}$ to be the coefficient of $b_{j}$ in $c_{i}$. As above, we calculate

$$
\begin{array}{r}
\alpha_{2 m}(6 m-l)=-\frac{6 m-l}{2 m} \sum_{\substack{1 \leq k \leq l-1,2 m-1 \\
k=\bmod 2=1}}\left(\begin{array}{c}
l-1 \\
k
\end{array}\right) \\
\cdot\left(\begin{array}{c}
(6 m-k-3) / 2 \\
2 m-1
\end{array}\right) .
\end{array}
$$

For $1 \leq l \leq 7$ and $m \geq 2$, or $1 \leq l \leq 5$ and $m \geq 1$, each term in the sum is nonnegative, so we can conclude that $\alpha_{2 m}(6 m-l) \leq 0$, with equality only when $l=1$. Similarly, $\alpha_{2 m+1}(6 m-1)<0$.

Also

$$
\alpha_{2 m}(6 m)=\left(\begin{array}{l}
3 m \\
2 m
\end{array}\right)
$$

and

$$
\alpha_{2 m+1}(6 m)=-8\left(\begin{array}{c}
3 m \\
2 m+1
\end{array}\right) \text {. }
$$

Finally,

$$
\beta_{i j}(n)=(-1)^{i} 2^{3 i-n}\left(\begin{array}{c}
k-j \\
i
\end{array}\right) .
$$

In particular,

$$
\beta_{(2 m+1) 0}(6 m)=\alpha_{2 m+1}(6 m), \beta_{(2 m) 0}(6 m)=\alpha_{2 m}(6 m)
$$

and

$$
\begin{aligned}
& \alpha_{2 m+1} \beta_{(2 m) j}-\alpha_{2 m} \beta_{(2 m+1) j} \\
& =\frac{8 m j}{(2 m+1)(2 m-j+1)}\left(\begin{array}{c}
3 m-j \\
m
\end{array}\right)\left(\begin{array}{c}
3 m \\
m
\end{array}\right) \geq 0
\end{aligned}
$$

with equality only when $j=0$.

We can now prove the following

Theorem 6: If a self-dual $[6 m+l, 6 m+l, d]_{4}$ exists, with $0 \leq l \leq 5$, then

$$
d \leq \begin{cases}2 m+2, & l<5 \\ 2 m+3, & l=5\end{cases}
$$

If a self-dual $[6 m+5,6 m+5, d]_{4}$ exists, then so does an even self-dual $[6 m+6,6 m+6, d]_{4}$. Finally, any self-dual $[6 m+6,6 m+6, d]_{4}$ must be even.

Proof: Proof as before. We need only give a construction of a $[6 m+6,6 m+6,2 m+4]_{4}$ from a $[6 m+5,6 m+5,2 m+3]_{4}$. Letting $C^{(i)}$ for $0 \leq i \leq 3$ be the four cosets of the even subcode $C^{(0)}$ in its dual, we can construct an even self-dual $[6 m+6,6 m+6,2 m+4]_{4}$ as the set of all vectors of one of the following forms: $0 \mid v$, for $v \in C^{(0)}, 1 \mid v$, for $v \in C^{(1)}, \omega \mid v$, for $v \in C^{(2)}$, and $\omega^{2} \mid v$, for $v \in C^{(3)}$.

\section{Self-Orthogonal Additive Codes}

For applications to quantum error-correcting codes, the objects of interest are additive codes $C$ over GF(4), selforthogonal under the trace-Hermitian inner product. In particular, we would like a bound on the minimum weight of $C^{\perp}-C$, given that $C$ has length $n$ and dimension $n-r<n$. (If $r=0$, then $C^{\perp}-C$ is empty.) If we merely wanted a bound on the minimum distance of $C^{\perp}$, we could simply apply Theorem 6 , since $C^{\perp}$ would contain some self-dual code; however, the problem as stated is not quite so simple.

Let $A(x, y), B(x, y)$, and $S(x, y)$ be the enumerators of $C, C^{\perp}$, and the shadow of $C$, respectively; then $B(x, y)-$ $A(x, y)$ is the weight enumerator of $C^{\perp}-C$. Thus we need to find a nonnegative linear combination of the coefficients of $B(x, y)-A(x, y), A(x, y)$, and $S(x, y)$ that equals 0 , producing a contradiction.

Note, first, that

$$
B(x, y)=2^{r} A((x+3 y) / 2,(x-y) / 2)
$$

so

$$
\begin{aligned}
\Delta(x, y) & \triangleq A(x, y)-A\left(\frac{x+3 y}{2}, \frac{x-y}{2}\right) \\
& =\left(1-2^{-r}\right) A(x, y)-2^{-r}(B(x, y)-A(x, y)) .
\end{aligned}
$$

In particular, since the first $d$ coefficients of $B(x, y)-A(x, y)$ are 0 (by assumption), we have

$$
\Delta(1, y)=\left(1-2^{-r}\right) A(1, y)+O\left(y^{d}\right)
$$

Note that

$$
\begin{aligned}
\Sigma(x, y) & \triangleq 2^{r-1} \Delta\left(\frac{x+3 y}{2}, \frac{y-x}{2}\right) \\
& =\frac{1}{2}(S(x, y)-S(-x, y))
\end{aligned}
$$

so $\Sigma(x, y)$ must have nonnegative coefficients.

What we will do, then, is produce a linear combination of the first $d$ coefficients of $\Delta(1, y)$ that is also a linear combination of certain coefficients of $\Sigma(1, y)$; again, the signs will give a contradiction. The main reason we can do this is the following theorem (analogous to Theorems 3 and 4 above).

Theorem 7: Let $\Delta(x, y)$ and $\Sigma(x, y)$ be as above. Then there exist coefficients $e_{i}, 0 \leq i \leq\lfloor(n-1) / 2\rfloor$, such that

$$
\begin{aligned}
& \Delta(x, y)=\sum_{0 \leq i \leq\lfloor(n-1) / 2\rfloor} e_{i}(x-3 y)(x+y)^{n-1-2 i}(y(x-y))^{i} \\
& \Sigma(x, y)=\sum_{0 \leq i \leq\lfloor(n-1) / 2\rfloor}(-1)^{i} 2^{n-1+r-3 i} e_{i} x y^{n-1-2 i}\left(x^{2}-y^{2}\right)^{i}
\end{aligned}
$$

Proof: Simply note that $\Delta(x, y)$ is taken to its negative by the MacWilliams transform

$$
\Delta\left(\frac{x+3 y}{2}, \frac{x-y}{2}\right)=-\Delta(x, y) .
$$

This follows from the fact that the substitution

$$
(x, y) \mapsto\left(\frac{x+3 y}{2}, \frac{x-y}{2}\right)
$$

is self-inverse.

This forces $\Delta(x, y)$ to be in the ring

$$
(x-3 y) \mathbb{C}[x+y, y(x-y)]
$$

One readily verifies that every element of this ring is antiinvariant under the MacWilliams transform; on the other hand, the Molien series of the ring of anti-invariants is $\frac{\lambda}{(1-\lambda)\left(1-\lambda^{2}\right)}$. Thus we have exhausted the space of anti-invariants.

The theorem follows immediately.

As one might expect, the linear combination we use will be a suitably chosen $e_{i}$. Let us therefore write $n=2 k+t+1$, 
with $t \in\{0,1\}$, and

$$
\begin{aligned}
& \Delta(1, y)=\sum_{0 \leq i \leq n} f_{i} y^{i} \\
& \Sigma(1, y)=\sum_{0 \leq i \leq k} g_{i} y^{2 i+t} .
\end{aligned}
$$

Let $\phi_{i j}$ be the coefficient of $f_{j}$ in the expansion of $e_{i}$ in terms of the $f_{j}$; similarly, let $\gamma_{i j}$ be the coefficient of $g_{j}$ in the expansion of $e_{i}$. Then we can compute $\phi_{i j}$ and $\gamma_{i j}$ by applying the Bürmann-Lagrange theorem to the identities

$y^{j}(1-3 y)^{-1}(1+y)^{-n+1}=\sum_{0 \leq i \leq k} \phi_{i j}\left(\frac{y(1-y)}{(1+y)^{2}}\right)^{i}+O\left(y^{k+1}\right)$

and

$$
\begin{aligned}
(-1)^{k} 2^{r+k-2-t} Y^{j} & (1-Y)^{-k} \\
& =\sum_{0 \leq i \leq k} \gamma_{(k-i) j}\left(\frac{-8 Y}{(1-Y)}\right)^{i}+O\left(Y^{k+1}\right)
\end{aligned}
$$

where $Y=y^{2}$.

Before applying the Bürmann-Lagrange theorem, it will be helpful to restate the theorem slightly.

Lemma 8: Let $f(x)$ and $g(x)$ be formal power series, with $g(0)=0$, and $g^{\prime}(0) \neq 0$. If coefficients $\kappa_{i j}$ are defined by

$$
x^{j} f(x)=\sum_{0 \leq i} \kappa_{i j} g(x)^{i}
$$

then

$$
\kappa_{i j}=\left[\text { coeff. of } x^{i-j} \text { in } \frac{x g^{\prime}(x)}{g(x)} f(x)\left(\frac{x}{g(x)}\right)^{i}\right] .
$$

Proof: The Bürmann-Lagrange theorem, as stated above, tells us that

$$
\begin{aligned}
\kappa_{i j} & =\frac{1}{i}\left[\text { coeff. of } x^{i-1} \text { in }\left(j x^{j-1} f(x)+x^{j} f^{\prime}(x)\right)\left(\frac{x}{g(x)}\right)^{i}\right] \\
& =\frac{1}{i}\left[\operatorname{coeff.~of~} x^{i-j} \text { in }\left(j+\frac{x f^{\prime}(x)}{f(x)}\right) f(x)\left(\frac{x}{g(x)}\right)^{i}\right] .
\end{aligned}
$$

Now, for any function $h(x)$

$$
\text { [coeff. of } \left.x^{i-j} \text { in }(i-j) h(x)-x h^{\prime}(x)\right]=0 .
$$

Applying this when $h(x)=f(x)(x / g(x))^{i}$, and adding into $\kappa_{i j}$, we get the desired result.

In particular

$$
\phi_{i j}=\left[\text { coeff. of } y^{i-j} \text { in }(1+y)^{2 i-n}(1-y)^{-i-1}\right] .
$$

Thus taking $n=6 m-l$ as before

$$
\left.\phi_{(2 m-1) j}=\text { [coeff. of } y^{2 m-1-j} \text { in }(1+y)^{l-2}\left(1-y^{2}\right)^{-2 m}\right] \text {. }
$$

This is positive whenever $l>2$; for $l=2$, it is nonnegative, and 0 only when $j$ is even. We also have, for $l=2$,

$$
\phi_{(2 m) j}=\left[\text { coeff. of } y^{2 m-j} \text { in }(1+y)^{3}\left(1-y^{2}\right)^{-2 m-1}\right]>0 .
$$

For $l=1$

$$
\begin{aligned}
\phi_{(2 m-1) j} & =\left[\text { coeff. of } y^{2 m-1-j} \text { in }(1-y)\left(1-y^{2}\right)^{-2 m-1}\right] \\
& =(-1)^{j+1}\left(\begin{array}{c}
3 m-1-\left\lfloor\frac{j}{2}\right\rfloor \\
2 m
\end{array}\right)
\end{aligned}
$$

and

$$
\begin{aligned}
\phi_{(2 m) j}= & {\left[\text { coeff. of } y^{2 m-j} \text { in }(1+y)^{2}\left(1-y^{2}\right)^{-2 m-1}\right] } \\
& = \begin{cases}\frac{2 m-\left\lfloor\frac{j}{2}\right\rfloor}{m}\left(\begin{array}{c}
3 m-\left\lfloor\frac{j}{2}\right\rfloor-1 \\
2 m-1
\end{array}\right), & j \text { even } \\
2\left(\begin{array}{c}
3 m-\left\lfloor\frac{j}{2}\right\rfloor-1 \\
2 m
\end{array}\right), & j \text { odd. }\end{cases}
\end{aligned}
$$

In particular

$$
\phi_{(2 m) j}+4 \phi_{(2 m-1) j}=\left\{\begin{array}{cc}
\frac{2\left\lfloor\frac{j}{2}\right\rfloor}{3 m-\left\lfloor\frac{j}{2}\right\rfloor}\left(\begin{array}{c}
\left.3 m-\left\lfloor\frac{j}{2}\right\rfloor\right), \\
2 m
\end{array}\right) & j \text { even } \\
6\left(\begin{array}{c}
3 m-1-\left\lfloor\frac{j}{2}\right\rfloor \\
2 m
\end{array}\right), & j \text { odd. }
\end{array}\right.
$$

This is nonnegative, and 0 only when $j=0$.

Similarly, we can compute $\gamma_{i j}$

$$
\begin{aligned}
& \gamma_{(k-i) j}=2^{-r+1}\left[\text { coeff. of } Y^{i-j}\right. \text { in } \\
& \left.\qquad(-1)^{k-i} 2^{k-t-1-3 i}(1-Y)^{-1-k+i}\right] .
\end{aligned}
$$

So

$$
\begin{aligned}
\gamma_{i j} & =(-1)^{i} 2^{3 i-n-r+1}\left[\text { coeff. of } Y^{k-i-j} \text { in }(1-Y)^{-1-i}\right] \\
& =(-1)^{i} 2^{3 i-n-r+1}\left(\begin{array}{c}
k-j \\
i
\end{array}\right) .
\end{aligned}
$$

In particular, this is negative for $i$ odd; furthermore, for $l=1$

$$
\gamma_{(2 m) j}+4 \gamma_{(2 m-1) j}=-2^{-r+1} \frac{j}{m}\left(\begin{array}{c}
k-j \\
k-m
\end{array}\right)<0
$$

except when $j=0$. Also, for $l=1$

$$
\gamma_{(2 m) 0}=2^{-r+1}\left(\begin{array}{c}
3 m-1 \\
2 m-1
\end{array}\right)=2^{-r} \phi_{(2 m) 0} .
$$

We now have the inequalities necessary to prove

Theorem 9: Let $C$ be an additive code over GF (4), of length $n=6 m-1+l$ with $0 \leq l \leq 5$, and dimension $n-r<n$, such that $C^{\perp}-C$ has minimum weight $d$. Then $d \leq 2 m+1$, except when $l=5$, when $d \leq 2 m+2$. Any code meeting the bound for $l=0$ must be the even subcode of a $[6 m-1,6 m-1,2 m+1]_{4}$.

Proof: For $1 \leq l \leq 4$, we have $\phi_{(2 m+1) j}>0$ and $\gamma_{(2 m+1) j}<0$, giving a contradiction. For $l=5$, we have $\phi_{(2 m+1) j}=0$ when $j$ is even; consequently, we can conclude only that $f_{j}=0$ for odd $j<(2 m+1)$, and that $g_{j}=0$ for all $j<m$. Now, consider $e_{2 m+2}$. This is a linear combination of the $g_{j}$ for $j<m-1$, so must equal 0 . On the other hand, it is also a positive linear combination of $f_{j}$ for $0 \leq j \leq 2 m+2$; this is impossible unless $d<2 m+2$.

Finally, for $l=0$, we consider $e_{2 m}+4 e_{2 m-1}$. This is a positive linear combination of $f_{j}$ for $1 \leq j \leq 2 m$, and a negative linear combination of $g_{j}$ for $1 \leq j \leq m$. Consequently, 
all of these $f_{j}$ and $g_{j}$ must be 0 . Then, considering $e_{2 m}$, we have

$$
e_{2 m}=\gamma_{(2 m) 0} g_{0}=\phi_{(2 m) 0} f_{0}
$$

so

$$
g_{0}=2^{r} f_{0}=2^{r}-1 .
$$

If $r>1$, then $g_{0}>1$, which is impossible (since $g_{0}=S(1,0)$ ); thus we must have $r=1$, so $g_{0}=1$ and the code is even. Clearly, then, if we take $D$ to be any self-dual code lying between $C$ and $C^{\perp}$, then $D$ must be a $[6 m-1,6 m-1,2 m+1]_{4}$, and $C$ is its even subcode. The theorem follows.

\section{FURTHER DIRECTIONS}

There is still some room for improvements in the above bounds. For instance, integer programming readily shows that no self-dual binary code of length 26 can meet the bound. It should be possible to systematize such effects by considering certain congruences modulo small powers of 2 in the coefficients of the weight and shadow enumerators. Also, it should be possible to show that only a finite number of codes can meet the bound, by considering $a_{4 m+8}$; in general, one would like a result saying that any bound of the form $n / 6-c$ can be exceeded only a finite number of times (this is known for doubly-even codes).

For self-orthogonal additive codes, the bound we give makes no use of the dimension of the code; for smaller codes, one ought to be able to produce much stronger bounds. It should be noted that one could prove a similar result for self-orthogonal binary codes that contain a vector of full weight; however, the object $C^{\perp}-C$ is much less natural in that case.

The theory of shadows also has an analogue for integral lattices [3]; as one might expect, therefore, the above bounds have analogues for lattices as well. For more details, consult [6].

\section{ACKNOWLEDGMENT}

The author wishes to thank N. Sloane for many productive conversations; in particular, for introducing the author to shadow theory.

\section{REFERENCES}

[1] A. R. Calderbank, E. M. Rains, P. W. Shor, and N. J. A. Sloane, "Quantum error correction via codes over GF (4)," LANL e-print quant$\mathrm{ph} / 9608006$.

[2] J. H. Conway and N. J. A. Sloane, "A new upper bound on the minimal distance of self-dual codes," IEEE Trans. Inform. Theory, vol. 36, no. 6, 1990.

[3] , "A new upper bound for the minimum of an integral lattice of determinant 1," Bull. Amer. Math. Soc., vol. 23, no. 2, 1990.

[4] S. T. Dougherty and M. Harada, "Extremal and shadow extremal binary self-dual codes," preprint.

[5] C. L. Mallows and N. J. A. Sloane, "An upper bound for self-dual codes," Inform. Contr., vol. 22, pp. 188-200, 1973.

[6] E. M. Rains and N. J. A. Sloane, "The shadow theory of modular and unimodular lattices," in preparation.

[7] H. N. Ward, "A bound for divisible codes," IEEE Trans. Inform. Theory, vol. 38, no. 1, pp. 191-195, 1992.

[8] E. T. Whittaker and G. N. Watson, A Course of Modern Analysis, 4th ed. New York: Cambridge Univ. Press, 1963. 